\title{
DEGRADABILIDAD RUMINAL DE LA FIBRA DEL FOLLAJE PISONAY (ERYTHRINA SP)
}

\section{RUMINAL DEGRADABILITY FIBER FOLIAGE OF PISONAY (ERYTHRINA SP)}

Ludwing Angel Cárdenas Villanueva ${ }^{1}$; José Luis Bautista Pampa ${ }^{2}$; Jorge Luis Zegarra

\section{Paredes $^{3}$; Ruth Ramos Zuñiga ${ }^{4}$}

${ }^{1}$ Facultad de Medicina Veterinaria y Zootecnia, Universidad Nacional Micaela Bastidas, Abancay.*E-mail: car-vet@hotmail.com.

${ }^{2}$ Facultad de Medicina Veterinaria y Zootecnia, Universidad Nacional del Altiplano, Puno. ${ }^{3}$ Laboratorio de Nutrición y Alimentación Animal de la Universidad Católica de Santa María de Arequipa.

${ }^{4}$ Práctica privada.

\section{RESUMEN}

El objetivo de este estudio fue evaluar la degradabilidad ruminal de la pared celular y sus fracciones a 105, 120 y 135 días de rebrote en hojas y peciolos de pisonay. Para estimar la degradabilidad se realizaron muestreos a las $0,24,48$ y 72 horas de incubación en tres machos cabríos canulados en el rumen y los datos se analizaron con un diseño experimental completamente al azar. La fibra detergente neutro presentó una fracción degradable de $42 \%$, $29 \%$ y $20 \%$ a 105,120 y 135 días, respectivamente, con tasas de degradación de 3\%. La degradación de la fibra detergente neutro se inició en el rumen con un tiempo de rezago de 4 a 9.3 horas. Se demuestra que este follaje debe ser consumido antes de los 120 días al observarse un mayor aprovechamiento por el animal.

Palabras clave: Composición química, cinética ruminal, cabras, hojas y peciolos

\section{ABSTRACT}

The aim of this study was ruminal degradability value of wall cellular and their fractions at 105,120 and 135 days of sprout in leaves and leafstalk. To evaluate the degradability was 
made samplings to $0,24,48$ and 72 hours of incubation in tree ruminally fistulated goat's male and data were analyzed completely randomized design. The neutral detergent fiber had a degradable fraction of $42 \%, 29 \%$ and $20 \%$ on the 105 th, 120 th y 135 th days respectively, with degradation rates of $3 \%$. The degradation neutral detergent fiber began in the rumen with a lag time of 4 to 9.3 hours. One demonstrates that this foliage must be consumed before the 120 days when being observed a greater advantage by the animal.

Key words: Chemical composition, ruminal kinetics, goats, leaves and leafstalk

\section{INTRODUCCIÓN}

Las leguminosas y otros arbustos y árboles tienen una biomasa rica en nutrientes, específicamente proteínas, vitaminas y minerales, por lo que se pueden utilizar como suplementos en las dietas para los rumiantes y aumentar la ingestión y la digestibilidad de los forrajes de menor calidad (Delgado et al., 2001). El follaje de algunos árboles y arbustos tropicales empleados en la alimentación de rumiantes se caracterizan por su aporte de nutrientes al ecosistema ruminal, fundamentalmente energía y nitrógeno; aunque la contribución a las partes bajas del tracto gastrointestinal de algunas especies leguminosas no es despreciable.

A raíz del déficit alimentario y la crisis económica mundial, los países latinoamericanos han tenido que incursionar en otras estrategias de alimentación para incrementar la producción animal (García y Medina, 2006). Actualmente se utiliza en menor medida la Erythrina sp considerada como una leguminosa arbustiva más usada como forraje (Shelton, 2000) que los productores cortan su follaje para alimentar a sus animales (Zamora et al., 2001). Los modelos actuales de alimentación recomiendan un mínimo de fibra para asegurar un correcto funcionamiento ruminal, porque aporta relativamente poca energía al animal y genera poca cantidad de ácidos grasos en el rumen, por otro lado aporta una textura física al contenido ruminal que estimula la rumia, la masticación y la secreción salival, y regula el ritmo de paso (Bach y Calsamiglia, 2006).

La estimación de la degradabilidad in situ, tiene como objetivo evaluar algunas características como la tasa y magnitud de la ingestión de alimentos, las cuales están relacionadas con la calidad nutritiva de los forrajes y puede dar un indicativo del aporte de nutrientes de las diferentes fuentes alimenticias utilizadas en los rumiantes. El objetivo del 
presente trabajo fue determinar las características degradativas de la pared celular de hojas y peciolos del pisonay.

\section{MATERIALES Y MÉTODOS}

El experimento se realizó en las instalaciones de la Universidad Nacional Micaela Bastidas, ubicado en el distrito de Tamburco, provincia Abancay, región Apurímac, a una altitud de $2581 \mathrm{~m}$, con una temperatura máxima media anual de $23,8^{\circ} \mathrm{C}$ y una mínima de $11,7^{\circ} \mathrm{C}$. La humedad relativa varía entre 59-70\%. La precipitación anual varía de 641 a 1119 $\mathrm{mm} / \mathrm{año.}$

Para determinar la composición química y degradabilidad ruminal se utilizaron hojas y peciolos de la Erythrina sp (pisonay) a 105, 120 y 135 días de rebrote. La fibra detergente neutro (FDN), fibra detergente acida (FDA) y lignina detergente ácido (LDA) se determinaron de acuerdo al método descrito por Van Soest et al. (1991), la hemicelulosa (HEM) por diferencia entre FDN - FDA y la celulosa (CEL) entre FDA - LDA.

Se realizó la intervención quirúrgica en tres machos cabríos adultos enteros con un peso vivo de $75.0 \pm 8.0 \mathrm{Kg}$ a nivel ruminal con la finalidad de fistular e instalar las cánulas permanentes (Bautista, 2004). Los animales 30 días antes y durante la etapa experimental se mantuvieron a pastoreo con Pennisetum clandestinum (pasto kikuyo) mas forraje fresco de pisonay en una cantidad de $2 \mathrm{Kg} /$ animal/día. El análisis de muestras se realizó en el Laboratorio de Nutrición y Alimentación Animal de la UCSM de Arequipa.

La degradabilidad ruminal del pisonay se estimó mediante el procedimiento de incubación en bolsas de nylon en el rumen (Mehrez y Orskov, 1977). Se incubaron 8 g de forraje molido por bolsa a $0,24,48$ y 72 horas. La cinética de degradación del pisonay se determinó con el modelo: $\mathrm{P}=\mathrm{a}+\mathrm{b}\left(1-\mathrm{e}^{-\mathrm{ct}}\right)$ y la degradabilidad efectiva: $\mathrm{DE}=\mathrm{a}+[(\mathrm{b} \times \mathrm{c}) /(\mathrm{c}$ + k)] (Orskov y McDonald, 1979). Para la tasa de pasaje (k) se utilizó 0.044/h (NRC, 1989) y 0.06/h (Michalet-Doreau et al., 1987). Para el análisis de los datos se utilizó un diseño completamente al azar con el PROC GLM del SAS. Para la comparación de medias se utilizó la prueba de rangos múltiples de Duncan. 


\section{RESULTADOS}

La composición química obtenida en la Erythrina sp a los 105, 120 y 130 días de rebrote se observan en la tabla $1(\mathrm{P} \leq 0.05)$, la MS se incrementa hasta $2.8 \%$ por efecto de los días de rebrote, la FDN, HEM y CEL fue similar a los 105 y 135 días. La FDA y LDA es mayor a los 120 días y menor a los 105 días, este comportamiento se debe probablemente a que las hojas presentan un mayor tamaño y tiene un menor número de inervaciones.

Tabla 1. Composición química de las hojas y peciolos de pisonay en tres edades de rebrote (días)

\begin{tabular}{ccccccc}
\hline $\begin{array}{c}\text { Edad de rebrote } \\
\text { (días) }\end{array}$ & MS & FDN & FDA & LDA & HEM & CEL \\
\cline { 2 - 7 } & $24.4 \mathrm{~b}$ & $51.2 \mathrm{~b}$ & $30.9 \mathrm{c}$ & $7.8 \mathrm{c}$ & $20.3 \mathrm{~b}$ & $23.2 \mathrm{~b}$ \\
105 & $24.8 \mathrm{~b}$ & $57.7 \mathrm{a}$ & $35.9 \mathrm{a}$ & $11.4 \mathrm{a}$ & $21.8 \mathrm{a}$ & $24.5 \mathrm{a}$ \\
120 & $27.2^{\mathrm{a}}$ & $52.5 \mathrm{~b}$ & $32.4 \mathrm{~b}$ & $9.3 \mathrm{~b}$ & $20.1 \mathrm{~b}$ & $23.1 \mathrm{~b}$ \\
135 &
\end{tabular}

$\mathrm{a}, \mathrm{b}, \mathrm{c}$, en una misma columna, señala diferencias, Duncan $(\mathrm{P} \leq 0.05)$

Los valores de la degradabilidad ruminal de la pared celular y de las diferentes fracciones fibrosas, se presentan en la tabla 2. La fracción potencialmente degradable de la FDN fue de $42 \%$ a los 105 días y disminuyo $(\mathrm{P} \leq 0.05)$ a $21 \%$ a los 135 días de edad del rebrote, este proceso necesitó 4 a 9 h para iniciar la degradación con una tasa de pasaje de $3.6 \% / \mathrm{h}$ en hojas y peciolos de 105 días y de $3.2 \% / \mathrm{h}$ a mayor edad. La FDA, fue similar en la fracción degradable entre 105 y 120 rebrotes, la cual fue degradada en forma constante a 11.4$13.6 \% / \mathrm{h}$ y resultó ser indegradable a 135 días de rebrote. La fracción degradable de la hemicelulosa fue de 55 a 32\% entre las edades de 105 y 135 días $(\mathrm{P} \leq 0.05)$ y se degradó a una velocidad de 5 y $1 \% / \mathrm{h}$, respectivamente, este proceso de degradación necesitó hasta una hora de reposo ruminal. La celulosa contenida en la FDA, presentó una fracción degradable distinta $(\mathrm{P} \leq 0.05)$ entre rebrotes. La degradabilidad efectiva de la FDN y HEM disminuyó conforme se incrementó la edad de rebrote y fue similar para la FDA. 
Tabla 2. Cinética ruminal y degradabilidad efectiva de las hojas y pecíolos de pisonay en tres edades de rebrote

\begin{tabular}{|c|c|c|c|c|c|c|c|c|}
\hline \multirow[t]{2}{*}{ Componente } & \multirow{2}{*}{$\begin{array}{l}\text { Edad de } \\
\text { rebrote } \\
\text { días }\end{array}$} & \multirow{2}{*}{$\begin{array}{l}\text { Valor } \\
\text { de a } \\
(\%)\end{array}$} & \multirow{2}{*}{$\begin{array}{l}\text { Valor } \\
\text { de b } \\
(\%)\end{array}$} & \multirow{2}{*}{$\begin{array}{l}\text { Valor } \\
\text { de c } \\
(\% / h)\end{array}$} & \multirow{2}{*}{$\begin{array}{l}\text { Tiempo } \\
\text { rezago } \\
\text { (h) }\end{array}$} & \multirow{2}{*}{$\begin{array}{c}\text { Sin } \\
\text { degradar } \\
(\%)\end{array}$} & \multicolumn{2}{|c|}{$\begin{array}{c}\text { Degradabilidad } \\
\text { Efectiva }\end{array}$} \\
\hline & & & & & & & $4.4 \% / h$ & $6.0 \% / h$ \\
\hline \multirow[t]{3}{*}{$\mathrm{MS}$} & 105 & $23.56 b$ & $35.71 \mathrm{a}$ & $4.42 \mathrm{a}$ & & 40.73 & $41.46 a$ & $38.71 \mathrm{a}$ \\
\hline & 120 & $22.94 c$ & $26.45 b$ & $4.30 \mathrm{~b}$ & & 50.61 & $36.01 \mathrm{~b}$ & $33.98 b$ \\
\hline & 135 & $24.63 a$ & $18.17 \mathrm{c}$ & $4.13 c$ & & 57.20 & $33.43 \mathrm{c}$ & $32.04 \mathrm{c}$ \\
\hline \multirow[t]{3}{*}{ FDN } & 105 & 0.00 & $42.42 \mathrm{a}$ & $3.63 \mathrm{a}$ & 4.00 & 57.58 & $19.18 \mathrm{a}$ & $15.99 \mathrm{a}$ \\
\hline & 120 & 0.00 & $29.48 b$ & $3.36 \mathrm{~b}$ & 4.42 & 70.52 & $12.76 \mathrm{~b}$ & $10.58 b$ \\
\hline & 135 & 0.00 & $20.74 b$ & $3.20 \mathrm{~b}$ & 9.30 & 79.26 & $8.73 c$ & $7.21 \mathrm{c}$ \\
\hline \multirow[t]{3}{*}{ FDA } & 105 & 0.00 & $13.91 \mathrm{a}$ & $1.11 \mathrm{a}$ & 11.44 & 86.09 & $2.80 \mathrm{a}$ & $2.17 \mathrm{a}$ \\
\hline & 120 & 0.00 & $12.07 \mathrm{a}$ & $1.06 \mathrm{a}$ & 13.04 & 87.93 & $2.34 \mathrm{a}$ & $1.81 \mathrm{a}$ \\
\hline & 135 & 0.00 & 0.00 & 0.00 & & 100.00 & & \\
\hline \multirow[t]{3}{*}{ HEM } & 105 & 0.00 & $54.94 \mathrm{a}$ & $4.76 \mathrm{a}$ & 0.10 & 45.06 & $28.55 \mathrm{a}$ & $24.30 \mathrm{a}$ \\
\hline & 120 & 0.00 & $41.65 b$ & $3.38 b$ & 0.25 & 58.35 & $19.30 \mathrm{~b}$ & $16.15 b$ \\
\hline & 135 & 0.00 & $31.65 \mathrm{c}$ & $1.21 \mathrm{c}$ & 1.16 & 68.35 & $6.83 c$ & $5.31 \mathrm{c}$ \\
\hline \multirow[t]{3}{*}{ CEL } & 105 & 0.00 & $23.80 \mathrm{a}$ & $1.56 \mathrm{a}$ & 1.12 & 76.20 & $6.23 \mathrm{a}$ & $4.91 \mathrm{a}$ \\
\hline & 120 & 0.00 & $19.54 b$ & $1.43 \mathrm{~b}$ & 1.40 & 80.46 & $4.79 b$ & $3.76 b$ \\
\hline & 135 & 0.00 & 0.00 & 0.00 & & 100.00 & & \\
\hline
\end{tabular}

$\mathrm{a}, \mathrm{b}, \mathrm{c}$, en una misma columna, señala diferencias, Duncan $(\mathrm{P} \leq 0.05)$

La cinética de degradación ruminal en hojas y peciolos, en cada edad de rebrote, se observa en la Figura 1. 


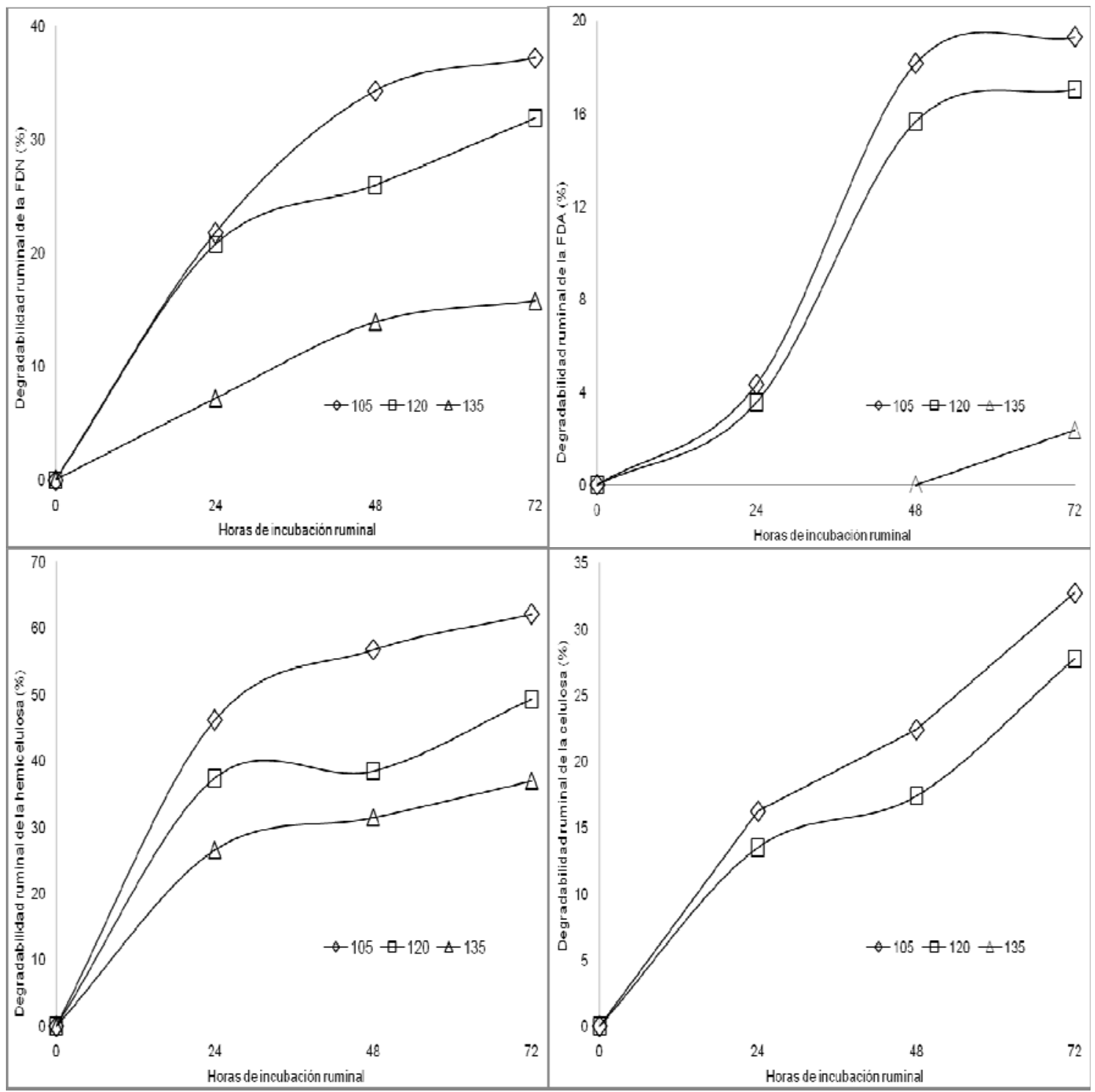

Figura 1. Degradabilidad ruminal de la FDN, FDA, HEM y CEL en tres edades de rebrote

\section{DISCUSIÓN}

Las diferencias numéricas encontradas en los componentes de la fracción fibrosa disminuyen y son similares entre 105 y 135 días. Al considerar los días de rebrote García et al. (2008) a 120 días evaluó seis arboles tropicales en la cual destacaron las leguminosa $E$. contortisilicum y L. latisiliquum (FDN: 49.9 y 48.8\%; FDA: 23.1 y 32.1\%), García et al. (2006) a 180 días en la biomasa de leguminosas de los géneros Albizia, Cassia y Pithecellobium informaron valores para FDN: 39.5-45.1\%, FDA: 22.2-27.7\%, LDA: 9.614.5\%, HEM: 13.0-20.6\% y CEL: 6.8-9.8\%, estos niveles son inferiores al compararlos con nuestro estudio, debido al inicio de un nuevo rebrote secundario o diferencia entre especies, con estos valores podemos indicar que se debe cosechar el pisonay inclusive antes de los 105 días de rebrote. 
La degradabilidad del pisonay disminuyó conforme aumento la edad de la planta. Naranjo y Cuartas (2011) evaluaron la cinética de la degradación ruminal de la FDN del chachafruto (Erythrina edulis) e informaron valores para la fracción degradable de 43,94\% con una tasa de degradación 5.0\%/h. Estos valores son similares a los encontrados a 105 días de rebrote observados en este estudio.

\section{CONCLUSIONES}

La fibra del follaje de pisonay tiene valores altos de degradación ruminal a los 105 días. Se demuestra que este follaje debe ser consumido antes de los 120 días al observarse un mayor aprovechamiento por el animal.

\section{AGRADECIMIENTOS}

Especial gratitud a los alumnos del Circulo de Estudio para la Investigación (CESINU), Facultad de Medicina Veterinaria de la Universidad Nacional Micaela Bastidas de Apurímac, por su apoyo para realizar el presente trabajo de investigación.

\section{BIBLIOGRAFÍA}

Bach, A y Calsamiglia S. 2006. La fibra en los rumiantes: ¿química o física? XXII Curso de especialización FEDNA. 16 p.

Bautista, JL. 2004. Desarrollo de cánulas en animales fistulados en el sistema digestivo. FMVZ. UNA, Puno, Perú.

Delgado, DC, La O, O, Chongo, B, Galindo, J, Obregón, Y y Aldama AI. 2001. Cinética de la degradación ruminal in situ de cuatro árboles forrajeros tropicales: Leucaena leucocephala, Enterolobium cyclocarpum, Sapindus saponaria y Gliricidia sepium. Revista Cubana de Ciencia Agrícola 35(2):141-145.

García, DE y Medina MG. 2006. Composición química, metabolitos secundarios, valor nutritivo y aceptabilidad relativa de diez árboles forrajeros. Zootecnia Trop. 24(3):233250.

García, DE, Medina, MG, Humbría, J, Domínguez, C, Baldizán, A, Coval L y Soca M. 2006. Composición proximal, niveles de metabolitos secundarios y valor nutritivo del follaje de algunos árboles forrajeros tropicales. Arch. Zootec. 55(212):373-384.

García, DE, Medina, MG, Clavero, T, Cova, LJ, Domínguez C y Baldizán A. 2008. Caracterización nutritiva del follaje de seis especies forrajeras con énfasis en sus perfiles polifenólicos. Revista Científica, FCV-LUZ 18(2):188-196. 
Mehrez, AZ y Orskov ER. 1977. A study of the artificial fibre bag technique for determining the digestibility of feeds in the rumen. J. Agric. Sci. 88:645-650.

Michalet-doreau, B, Verite, R y Chapoutot. 1987. Méthodologie de mesure de la degrdabilite in sacco de I'azote des aliments dans le rumen. Bull tech, Cent. Rech. Zootech Vet Theix. INRA 69:5-7.

Naranjo, JF y Cuartas CA. 2011. Caracterización nutricional y de la cinética de degradación ruminal de algunos de los recursos forrajeros con potencial para la suplementación de rumiantes en el trópico alto de Colombia. Rev CES Med Vet Zootec. 6 (1):9-19.

National Research Council. 1989. Nutrient requirements of dairy cattle. $6^{\text {th }}$ edited of National Academy of Science. Washington, D.C. 157 p.

Orskov, ER y McDonald I. 1979. The estimation of protein degradability in the rumen from incubation measurements weighted according to rate of passage. J. Agric. Sci., Camb. 92:499-503.

Shelton, M. 2000. Leguminosas forrajeras tropicales en los sistemas agroforestales. Unasylva 51: $25-32$.

Van Soest, PJ, Robertson, J y Lewis B. 1991. Methods for dietary fiber, neutral detergent fiber and nonstarch polysaccharides in relation to animal nutrition. J. Dairy Sci. 74:35833597.

Zamora, S, García, J, Bonilla, G, Aguilar, H, Harvey, CA y Ibrahim M. 2001. Uso de frutos y follaje arbóreo en la alimentación de vacunos en la época seca en Boaco, Nicaragua. Agroforestería en las Américas 8(31):31-38. 